\title{
Consumo, digestibilidade, desempenho, desenvolvimento ponderal e economicidade de dietas com polpa cítrica em substituição ao feno de capim-tifton 85 para novilhas leiteiras ${ }^{1}$
}

\section{Josué Mendes Neto ${ }^{2}$, José Maurício de Souza Campos ${ }^{3}$, Sebastião de Campos Valadares Filho $^{3}$, Rogério de Paula Lana ${ }^{3}$, Augusto César de Queiroz ${ }^{3}$, Ricardo Frederico Euclydes ${ }^{3}$}

\footnotetext{
1 Parte da tese do primeiro autor apresentada à Universidade Federal de Viçosa para obtenção do título de Magister Scientiae em Zootecnia. 2 Doutorando, DZO-UFV, Viçosa-MG, CEP: 36571-000.

${ }^{3}$ Departamento de Zootecnia, Universidade Federal de Viçosa, Viçosa-MG, CEP: 36571-000.
}

RESUMO - Objetivou-se determinar o consumo, a digestibilidade, o desempenho, o desenvolvimento ponderal, a conversão alimentar e a economicidade de dietas com polpa cítrica em substituição ao feno de tifton 85 (Cynodon dactylon (L.) Pear) para novilhas leiteiras. Os tratamentos consistiram de quatro níveis $(0 ; 16,6 ; 33,3$ e 50\%) de substituição do feno por polpa cítrica. Os dados foram analisados em delineamento experimental de blocos casualizados com sete repetições. Utilizaram-se 28 novilhas holandesas ( 12 meses de idade e peso médio inicial de $184 \mathrm{~kg}$ ) mantidas em baias individuais. Os consumos de MS, MO, PB, CT e NDT aumentaram linearmente e o de FDN decresceu à medida que o feno foi substituído pela polpa cítrica. O consumo de EE elevou de forma quadrática com aumento dessa substituição, apresentando valor mínimo quando 2,27\% do feno foi substituído pela polpa cítrica. Os consumos de Ca e P aumentaram com o aumento dos níveis de substituição do feno. Os coeficientes de digestibilidade aparente da MS, MO, PB e FDN reduziram linearmente, enquanto os de EE e CT não foram influenciados pela substituição. O ganho de peso aumentou linearmente, mas o crescimento de altura de cernelha foi menor e o de altura de garupa não foi influenciado pelos níveis de substituição. Observou-se aumento linear no perímetro torácico à medida que o feno foi substituído pela polpa cítrica. A polpa cítrica pode ser utilizada em até 35\% da dieta total na alimentação de novilhas leiteiras de acordo com a conveniência econômica e a disponibilidade de alimentos volumosos, como alternativa para o produtor reduzir a idade à primeira cobertura e ao primeiro parto, com menor custo de alimentação.

Palavras-chave: altura de cernelha, altura de garupa, carboidratos não-fibrosos, conversão alimentar, digestão de fibra, perímetro torácico

\section{Effects of partial replacement of Tifton 85 hay with citrus pulp on intake, performance, and development of dairy heifers}

\begin{abstract}
The objective of this trial was to evaluate intake, digestibility, performance, development, feed efficiency and feeding costs in dairy heifers fed increasing levels of citrus pulp that partially replaced Tifton-85 hay (Cynodon dactylon (L.) Pear) in the diet. Twenty-eight Holstein dairy heifers averaging 12 months of age and $184 \mathrm{~kg}$ of $\mathrm{BW}$ at the beginning of the trial were blocked by initial $\mathrm{BW}$ and, within each block (7 blocks), randomly assigned to one of the following four treatments: $0,16.6,33.3$, or $50 \%$ of a concentrate based on citrus pulp that partially replaced Tifton 85 hay in the diet. Intakes of DM, OM, CP,TCHO, TDN, Ca, and P all increased linearly while that of NDF was the opposite by partially replacing Tifton 85 hay with citrus pulp. A significant quadratic effect was observed for ether extract intake (EE) with quadratic minimum at $2.27 \%$ of hay replaced by citrus pulp. Apparent total tract digestibilities of DM, OM, CP, and NDF decreased linearly whereas that of EE and TCHO was not affected by replacing hay with citrus pulp in the diet. Although the average daily weight gain and heart girth increased linearly, the opposite was observed for wither height while hip height was not affected by replacing hay with citrus pulp. It was observed a linear increase in gross revenue and gross margin and a linear reduction in the cost per $\mathrm{kg}$ of weight gain on dairy heifers fed increasing levels of dietary citrus pulp. It can be concluded that up to $35 \%$ of citrus pulp can be included in the total diet DM of dairy heifers with significant reduction in the feeding costs.
\end{abstract}

Key Words: feeding efficiency, fiber digestion, hip height, heart girth, non fiber carbohydrates, wither height 


\section{Introdução}

A pecuária leiteira tem passado por profundas modificações para o aumento da eficiência de produção. Neste sentido, a criação de novilhas tem sido historicamente deixada em segundo plano, provavelmente porque o retorno financeiro ocorre a médio e longo prazo e porque os custos inerentes à criação são elevados. A criação de novilhas leiteiras tem sido apontada como a segunda maior fonte de despesas no sistema de produção. Segundo Sejrsen \& Purup (1997), a forma mais eficiente de redução no custo desta categoria seria a redução na idade ao primeiro parto, associada à redução no custo da alimentação, ao aumento do número de animais em produção e à maior liquidez do investimento. A polpa cítrica é considerada um concentrado energético e utilizada com freqüência como substituto ao milho para redução dos custos de produção (Fegeros et al., 1995). A utilização deste subproduto na alimentação de ruminantes é associada à manutenção das condições ruminais mais estáveis, com efeitos semelhantes aos observados quando se utilizam alimentos volumosos, e particularmente importante quando a dieta tem elevada relação concentrado:volumoso (Wing, 1982).

A polpa cítrica, por sua disponibilidade e seu preço de mercado, pode ser mais uma opção para alimentação de novilhas. No Brasil, estima-se que a produção de polpa cítrica na safra 1998-1999 chegou a um milhão de toneladas e que 70 a $75 \%$ deste montante foi exportado para países da Europa (Rocha Filho, 1998). A cotação histórica da polpa cítrica é de aproximadamente U\$ 100,00 por tonelada e a variação do preço depende da época do ano, da oferta e procura no mercado internacional e do preço do milho.

Van Soest (1994) e Bruno Filho et al. (2000) afirmaram que a digestibilidade da fração protéica da polpa cítrica pode ser reduzida pela influência do processo de secagem, principalmente a alta temperatura utilizada, causando redução na disponibilidade dos compostos nitrogenados, como resultado da relação de Maillard. No entanto, apesar de este processo diminuir a disponibilidade dos compostos nitrogenados, não foi observada alteração significativa no teor de PB das polpas cítricas estudadas. Este fato pode ser particularmente importante quando se utiliza polpa cítrica na alimentação de animais em crescimento.

Lammers \& Heinrichs (2000) verificaram, em novilhas, estreita correlação entre a relação proteína:energia das dietas e a redução na produção de leite ao longo da vida produtiva do animal quando submetido a elevadas taxas de crescimento. Segundo o NRC (2001), o requerimento de proteína depende principalmente do peso corporal do animal, do ganho de peso diário e da digestibilidade da proteína da dieta, sendo que a relação proteína:energia requerida pelos animais pode ser maior em novilhas submetidas a maiores taxas de crescimento. Portanto, a utilização indiscriminada de polpa cítrica na alimentação de novilhas leiteiras visando apenas à redução nos custos de alimentação pode resultar em diminuição da fertilidade e da produção de leite dos animais submetidos a elevadas taxas de crescimento.

Em regiões tropicais, observa-se grande estacionalidade na produção e qualidade da forragem. Desse modo, o produtor recorre a técnicas de conservação, como fenação, para garantir forragem de boa qualidade para os animais no período de maior escassez de alimentos. O preço do feno de tifton de boa qualidade na região da Zona da Mata de Minas Gerais é de aproximadamente U\$ 135,00 por tonelada e está sujeito a variações, como aumento da procura na época da estiagem.

A polpa cítrica poderia ser utilizada em substituição ao feno de tifton para novilhas leiteiras na tentativa de diminuir os custos com alimentação e aumentar a taxa de ganho de peso, diminuindo a idade ao primeiro parto e aumentando o retorno econômico da atividade.

O objetivo nesta pesquisa foi avaliar o efeito da substituição do feno de tifton 85 pela polpa cítrica sobre o consumo de nutrientes, a digestibilidade aparente dos nutrientes, o desempenho, o desenvolvimento ponderal, a conversão alimentar e a economicidade das dietas para novilhas leiteiras.

\section{Material e Métodos}

O experimento foi realizado na Unidade de Pesquisa, Ensino e Extensão em Gado de Leite (UEPE-GL) do Departamento de Zootecnia da Universidade Federal de Viçosa. Foram utilizadas 28 novilhas holandesas malhadas de preto, puras e mestiças, com grau de sangue variando de $7 / 8$ Holandês-Zebu a puro por cruza (12 meses de idade e peso inicial de $184 \mathrm{~kg}$ ). Os animais foram submetidos a tratamento com vermífugo, carrapaticida e complexo vitamínico ADE antes do início do período experimental. As novilhas foram alojadas em baias individuais cobertas, com $8 \mathrm{~m}^{2}$ de área (5,6 $\mathrm{m}^{2}$ com piso cimentadoe $2,4 \mathrm{~m}^{2}$ de cama), com comedouros individuais de concreto e bebedouros automáticos. Como cama, utilizou-se casca de café devidamente misturada a esterco bovino para evitar o consumo pelos animais.

Os tratamentos consistiram de quatro níveis de substituição $(0 ; 16,6 ; 33,3$ e 50\%) de feno de tifton 85 (Cynodon dactylon (L.) Pear) picado grosseiramente (3 a $5 \mathrm{~cm}$ ) por polpa cítrica moída. As dietas, isoprotéicas e com mesmo teor de NNP, foram formuladas segundo exigências 
nutricionais descritas pelo NRC (1989) para proporcionar ganho médio de $700 \mathrm{~g} /$ dia. A proporção dos ingredientes nas dietas experimentais é apresentada na Tabela 1 e a composição químico-bromatológica do feno, dos concentrados e das dietas, na Tabela 2.
$\mathrm{O}$ experimento constou de 15 dias de adaptação às dietas, ao manejo e às instalações e de três períodos experimentais de 28 dias, perfazendo 84 dias de coleta de dados. No início e no final do experimento e a cada 28 dias, após jejum de 12 horas com acesso irrestrito à água, os animais

Tabela 1 - Composição dos concentrados e das dietas experimentais, com base na MS

Table 1 - Ingredient composition of concentrate and experimental diets (\% of DM)

\begin{tabular}{|c|c|c|c|c|}
\hline \multirow[t]{2}{*}{$\begin{array}{l}\text { Ingrediente } \\
\text { Ingredient }\end{array}$} & \multicolumn{4}{|c|}{$\begin{array}{l}\text { Nível de substituição (\%) } \\
\text { Replacement level (\%) }\end{array}$} \\
\hline & 0 & 16,6 & 33,3 & 50 \\
\hline \multicolumn{5}{|l|}{$\begin{array}{l}\text { Composição do concentrado } \\
\text { Concentrate composition }\end{array}$} \\
\hline Polpa cítrica (Citrus pulp) (\%) & 0,00 & 27,95 & 43,73 & 53,82 \\
\hline Milho (Corn, ground) (\%) & 85,49 & 53,52 & 34,67 & 22,62 \\
\hline Farelo de soja (Soybean meal) (\%) & 6,67 & 4,80 & 3,75 & 3,08 \\
\hline Farelo de algodão (Cottonseed meal) (\%) & 0,00 & 9,39 & 14,93 & 18,49 \\
\hline Uréia (Urea) $(\%)$ & 1,00 & 0,72 & 0,56 & 0,46 \\
\hline Fosfato bicálcico (Dicalcium phosphate) (\%) & 4,42 & 2,63 & 1,59 & 0,90 \\
\hline Calcário calcítico (Limestone) (\%) & 1,06 & 0,00 & 0,00 & 0,00 \\
\hline Sal mineral (Mineral salt) $(\%)$ & 1,36 & 0,98 & 0,76 & 0,63 \\
\hline BHT $(\mathrm{g} / 100 \mathrm{~kg})$ & 3,00 & 3,00 & 3,00 & 3,00 \\
\hline \multicolumn{5}{|l|}{$\begin{array}{l}\text { Composição da dieta } \\
\text { Diet composition }\end{array}$} \\
\hline Feno de tifton 85 (Tifton 85 hay) (\%) & 70,00 & 58,34 & 46,66 & 35,00 \\
\hline Polpa cítrica (Citrus pulp) (\%) & 0 & 11,65 & 23,33 & 35,00 \\
\hline Milho (Corn, ground) (\%) & 25,65 & 22,30 & 18,49 & 14,71 \\
\hline Farelo de soja (Soybean meal) (\%) & 2,00 & 2,00 & 2,00 & 2,00 \\
\hline Farelo de algodão (Cottonseed meal) (\%) & 0 & 3,91 & 7,95 & 12,02 \\
\hline Uréia (Urea) (\%) & 0,30 & 0,30 & 0,30 & 0,30 \\
\hline Fosfato bicálcico (Dicalcium phosphate) (\%) & 1,33 & 1,10 & 0,85 & 0,58 \\
\hline Calcário calcítico (Limestone) (\%) & 0,32 & 0 & 0 & 0 \\
\hline Sal mineral (Mineral salt) (\%) & 0,41 & 0,41 & 0,41 & 0,41 \\
\hline BHT $(\mathrm{g} / 100 \mathrm{~kg})$ & 0,9 & 1,25 & 1,60 & 1,95 \\
\hline
\end{tabular}

Tabela 2 - Composição química (\%) do feno de tifton 85, dos concentrados e das dietas Table 2 - Chemial composition (\%) of Tifton 85 hay, concentrate and diets

\begin{tabular}{|c|c|c|c|c|c|c|c|c|c|c|}
\hline & \multirow[t]{3}{*}{$\begin{array}{l}\text { Polpa cítrica } \\
\text { Citrus pulp }\end{array}$} & \multirow[t]{3}{*}{$\begin{array}{l}\text { Feno } \\
\text { Hay }\end{array}$} & \multicolumn{8}{|c|}{$\begin{array}{l}\text { Nível de substituição (\%) } \\
\text { Replacement level (\%) }\end{array}$} \\
\hline & & & \multicolumn{4}{|c|}{$\begin{array}{l}\text { Concentrado } \\
\text { Concentrate }\end{array}$} & \multicolumn{4}{|c|}{$\begin{array}{l}\text { Dieta } \\
\text { Diet }\end{array}$} \\
\hline & & & 0 & 16,6 & 33,3 & 50 & 0 & 16,6 & 33,3 & 50 \\
\hline MS (DM) & 88,7 & 90,72 & 87,59 & 87,66 & 87,50 & 87,59 & 89,78 & 89,43 & 89,01 & 88,69 \\
\hline MO $(O M)$ & 93,15 & 93,08 & 91,44 & 92,01 & 92,88 & 92,89 & 92,59 & 92,63 & 92,97 & 92,96 \\
\hline $\mathrm{PB}(C P)$ & 7,50 & 14,41 & 13,48 & 13,74 & 13,89 & 13,99 & 14,13 & 14,13 & 14,13 & 14,13 \\
\hline $\mathrm{NNP}(N P N)^{1}$ & 21,31 & 21,15 & 22,78 & 22,59 & 22,20 & 21,94 & 21,62 & 21,74 & 21,70 & 21,66 \\
\hline NIDA $(A D I N)^{1}$ & 38,96 & 29,35 & - & - & - & - & - & - & - & - \\
\hline $\mathrm{EE}(E E)$ & 3,10 & 2,00 & 4,72 & 3,72 & 3,60 & 3,57 & 2,82 & 2,72 & 2,85 & 3,02 \\
\hline $\mathrm{CT}(T C)$ & 77,01 & 76,68 & 73,24 & 74,56 & 75,39 & 75,33 & 75,64 & 75,79 & 75,99 & 75,80 \\
\hline FDN (NDF) & 25,15 & 71,70 & 9,72 & 13,80 & 16,23 & 18,07 & 53,01 & 47,38 & 42,30 & 36,84 \\
\hline $\mathrm{CNF}(N F C)$ & 53,0 & 4,98 & 63,52 & 60,75 & 59,16 & 57,26 & 22,54 & 28,40 & 33,69 & 38,96 \\
\hline FDA $(A D F)$ & 22,7 & 31,56 & 1,69 & 5,55 & 6,45 & 9,50 & 22,60 & 20,64 & 18,25 & 17,22 \\
\hline HEM & 9,60 & 33,48 & 6,77 & 6,86 & 6,47 & 6,88 & 25,47 & 22,30 & 19,17 & 16,19 \\
\hline CEL & 16,81 & 27,21 & 2,12 & 5,05 & 6,76 & 8,73 & 19,68 & 17,90 & 16,37 & 15,20 \\
\hline LIG & 2,6 & 4,38 & 0,61 & 1,37 & 1,62 & 2,18 & 3,22 & 3,11 & 2,91 & 2,95 \\
\hline NDT $(T D N)$ & - & - & - & - & - & - & 73,85 & 72,96 & 72,68 & 71,16 \\
\hline Cinzas (Ash) & 11,02 & 6,92 & 8,56 & 7,99 & 7,12 & 7,11 & 7,41 & 7,37 & 7,03 & 7,04 \\
\hline $\mathrm{Ca}$ & 1,80 & 0,54 & 1,91 & 1,74 & 1,31 & 1,43 & 0,95 & 1,04 & 0,95 & 1,12 \\
\hline $\mathrm{P}$ & 0,09 & 0,24 & 1,39 & 1,20 & 0,80 & 0,83 & 0,58 & 0,64 & 0,56 & 0,63 \\
\hline
\end{tabular}

$1 \%$ do $\mathrm{N}$ total $(\%$ of total $\mathrm{N})$. 
foram pesados individualmente e submetidos à medição do perímetro torácico, da altura de cernelha e da altura de garupa.

A alimentação foi fornecida duas vezes ao dia (às 7 e 17h) em quantidade calculada para permitir aproximadamente $10 \%$ de sobras, com base na MS. As sobras foram retiradas semanalmente e amostradas, sendo devidamente armazenadas a $-5^{\circ} \mathrm{C}$. $\mathrm{O}$ feno e o concentrado foram amostrados diariamente e, ao final de cada período experimental, foi feita uma amostra composta de feno, concentrado e sobras de cada animal.

A excreção fecal foi estimada por meio do fornecimento diário de $10 \mathrm{~g}$ de óxido de cromo, em dose única, às $11 \mathrm{~h}$, durante 11 dias ( seis dias de adaptação e cinco de coleta de fezes). O óxido de cromo foi embalado em cápsulas de papel e fornecido diretamente à altura do esôfago por meio da utilização de sonda esofagiana. As fezes foram coletadas diretamente no reto às $8,10,12,14$ e $16 \mathrm{~h}$, respectivamente,

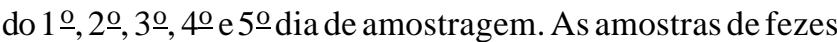
foram acondicionadas em sacos plásticos devidamente identificados e armazenadas em congelador a $-5^{\circ} \mathrm{C}$.

As amostras de fezes e de sobras dos alimentos foram pré-secas em estufa de circulação forçada a $65^{\circ} \mathrm{C}$. As amostras de feno, concentrado e sobras foram trituradas em moinho de faca tipo Wiley com peneiras de 30 mesh, sendo armazenadas em recipientes de vidro com tampa de polietileno para posteriores análises. As amostras de fezes foram descongeladas à temperatura ambiente e, em seguida, colocadas em bandejas de alumínio e submetidas à présecagem e processamento como descrito para as demais amostras. Posteriormente, foram separadas por animal e homogeneizadas para retirada de uma subamostra, com base no peso seco, para análises.

Os teores de MS, MO, NT e EE foram determinados segundo técnicas descritas por Silva (1990) e os FDN e FDA, segundo Van Soest et al. (1991). Os teores de cálcio e fósforo foram determinados na solução mineral, obtida pela digestão via úmida, sendo o $\mathrm{Ca}$ determinado por espectrofotomotria de absorção atômica e o $\mathrm{P}$ por colorimetria (Silva, 1990).

As análises do NNP foram realizadas segundo Licitra et al. (1996). Os CT foram estimados por meio da equação descrita por Sniffen et al. (1992): CT $=100-(\% \mathrm{~PB}+\% \mathrm{EE}+$ \% Cinzas) e os não-fibrosos (CNF), pela diferença entre carboidratos totais CT e FDN. O consumo de NDT (cNDT) foi determinado utilizando-se a fórmula descrita por Sniffen et al. $(1992), \mathrm{cNDT}=(\mathrm{cPB}-\mathrm{PBf})+2,25(\mathrm{cEE}-\mathrm{Eef})+(\mathrm{cCT}$ - CTf), em que cPB, cEE e cCT significam os consumos de PB, EE e CT, respectivamente, e PBf, EEf e CTf, os teores de
PB, EE e CT nas fezes. As análises de cromo nas fezes foram realizadas por meio de espectrofotometria de absorção atômica, de acordo com a técnica proposta por Williams et al. (1962). A economicidade foi determinada por meio de análises simples de custos com alimentação, receita bruta, margem bruta e custo da alimentação por quilograma de ganho de peso corporal.

Os dados de consumo de nutrientes, digestibilidade aparente dos nutrientes, desempenho, desenvolvimento ponderal e economicidade das dietas experimentais foram analisados em delineamento experimental de blocos casualizados, com quatro tratamentos (níveis de substituição do feno pela polpa cítrica), em que os animais foram distribuídos em sete blocos conforme seu peso inicial.

As análises estatísticas foram realizadas por meio do SAEG (Sistema de Análise Estatística e Genética) (UFV, 1995), segundo o modelo teórico:

$$
Y i j=\mu+t i+b j+e i j
$$

em que: $Y i j$ = valor observado na parcela que recebeu a dieta i e se encontra no bloco j; $\mu=$ média geral; $\mathrm{ti}=$ efeito do nível de substituição $i$, sendo $i=0,16,6,33,3$ e 50\%; bj = efeito do bloco j; e eij = efeito dos fatores não controlados dentro da parcela.

\section{Resultados e Discussão}

Constam na Tabela 3 as médias, as equações de regressão e os coeficientes de variação e determinação para os consumos de MS, MO, PB, EE, CT, FDN, NDT, cálcio e fósforo obtidos com os diferentes níveis de substituição do feno pela polpa cítrica. Os consumos de MS, MO, PB, CT e NDT aumentaram de forma linear $(\mathrm{P}<0,01)$ à medida que $\mathrm{O}$ feno foi substituído pela polpa cítrica. O consumo de FDN, no entanto, decresceu $(\mathrm{P}<0,01)$ com o aumento dos níveis de substituição do feno nas dietas. O aumento nos níveis de substituição ocasionou redução do teor de FDN das dietas, como resultado do menor teor de FDN da polpa cítrica em comparação ao feno. Além disso, a fração fibrosa da polpa cítrica é, segundo Van Soest (1994) e Carvalho \& Peres (1995), de rápida e elevada degradabilidade ruminal. Essas características podem ter aumentado a taxa de degradação ruminal da fração fibrosa das dietas, diminuindo o tempo de retenção e aumentando a taxa de passagem, o que explica o aumento do consumo de MS.

Neste estudo, a polpa cítrica foi finamente moída, de modo que a granulometria das partículas das dietas diminuiu com o aumento do nível de substituição. Com a diminuição do tamanho e o aumento da densidade das partículas, em comparação às partículas do feno $(3 \mathrm{a} 5 \mathrm{~cm})$, podem ter 
Tabela 3 - Consumos médios diários de nutrientes em novilhas alimentadas com dietas contendo polpa cítrica em substituição ao feno de tifton 85

Table 3 - $\quad$ Average daily nutrient intakes in dairy heifers fed diets containing increasing levels of citrus pulp in replacement of Tifton 85 hay

\begin{tabular}{|c|c|c|c|c|c|c|c|}
\hline & \multicolumn{4}{|c|}{$\begin{array}{c}\text { Nível de substituição (\%) } \\
\text { Replacement level (\%) }\end{array}$} & \multirow[t]{2}{*}{$\mathrm{CV}$} & \multicolumn{2}{|c|}{$\mathrm{P}$} \\
\hline & 0 & 16,6 & 33,3 & 50 & & $\mathrm{~L}$ & Q \\
\hline $\mathrm{MS}^{1}(\% \mathrm{PV})(D M, \% B W)$ & 2,64 & 2,78 & 2,91 & 3,05 & 4,01 & 0,0001 & ns \\
\hline $\mathrm{MS}^{2}\left(\mathrm{~g} / \mathrm{kg}^{0,75}\right)\left(D M, g / \mathrm{kg}^{0.75}\right)$ & 102,86 & 108,90 & 113,59 & 120,11 & 4,96 & 0,0001 & $\mathrm{~ns}$ \\
\hline $\mathrm{MS}^{3}(\mathrm{~kg} / \mathrm{dia})(D M, \mathrm{~kg} /$ day $)$ & 6,26 & 6,71 & 6,91 & 7,47 & 8,64 & 0,0012 & $\mathrm{~ns}$ \\
\hline $\mathrm{MO}^{4}(\% \mathrm{PV})(O M, \% B W)$ & 2,44 & 2,58 & 2,70 & 2,83 & 4,01 & 0,0001 & $\mathrm{~ns}$ \\
\hline $\mathrm{MO}^{5}\left(\mathrm{~g} / \mathrm{kg}^{0,75}\right)\left(O M, \mathrm{~g} / \mathrm{kg}^{0.75}\right)$ & 95,24 & 100,85 & 105,60 & 111,67 & 4,96 & 0,0001 & $\mathrm{~ns}$ \\
\hline $\mathrm{MO}^{6}(\mathrm{~kg} / \mathrm{dia})(O M, \mathrm{~kg} /$ day $)$ & 5,80 & 6,21 & 6,42 & 6,94 & 8,63 & 0,0009 & $\mathrm{~ns}$ \\
\hline $\mathrm{PB}^{7}$ (kg/dia) (CP, kg/day) & 0,89 & 0,95 & 0,98 & 1,06 & 8,64 & 0,0013 & $\mathrm{~ns}$ \\
\hline $\mathrm{EE}^{8}(\mathrm{~kg} / \mathrm{dia})(E E, \mathrm{~kg} /$ day $)$ & 0,18 & 0,18 & 0,20 & 0,23 & 8,51 & 0,0000 & 0,0592 \\
\hline $\mathrm{CT}^{9}{ }^{(\mathrm{kg} / \mathrm{dia})}(T C, \mathrm{~kg} /$ day $)$ & 4,73 & 5,08 & 5,25 & 5,66 & 8,64 & 0,0010 & $\mathrm{~ns}$ \\
\hline $\mathrm{FDN}^{10}(\% \mathrm{PV})(N D F, \% B W)$ & 1,38 & 1,31 & 1,22 & 1,12 & 4,66 & 0,0000 & $\mathrm{~ns}$ \\
\hline $\mathrm{FDN}^{11}\left(\mathrm{~g} / \mathrm{kg}^{0,75}\right)\left(N D F, g / \mathrm{kg}^{0.75}\right)$ & 53,81 & 51,25 & 47,77 & 44,10 & 5,63 & 0,0000 & ns \\
\hline $\mathrm{FDN}^{12}(\mathrm{~kg} / \mathrm{dia})(N D F, k g /$ day $)$ & 3,28 & 3,16 & 2,91 & 2,74 & 9,97 & 0,0024 & $\mathrm{~ns}$ \\
\hline $\mathrm{NDT}^{13}$ (kg/dia) $(T D N, \mathrm{~kg} /$ day $)$ & 5,25 & 4,95 & 5,70 & 6,06 & 9,17 & 0,0011 & $\mathrm{~ns}$ \\
\hline $\mathrm{Ca}^{14}(\mathrm{~g} / \mathrm{dia})(\mathrm{Ca}, \mathrm{g} /$ day $)$ & 61,59 & 71,80 & 66,77 & 84,23 & 8,59 & 0,0001 & 0,0766 \\
\hline $\mathrm{P}^{15}(\mathrm{~g} / \mathrm{dia})(P, g /$ day $)$ & 36,71 & 43,33 & 38,89 & 47,14 & 8,03 & 0,0015 & $\mathrm{~ns}$ \\
\hline
\end{tabular}

${ }^{1} \hat{Y}=2,64190+0,00810936 X\left(r^{2}=0,99\right) ;{ }^{2} \hat{Y}=108,889+0,338838 X\left(r^{2}=0,99\right) ;{ }^{3} \hat{Y}=6,25835+0,0229832 X\left(r^{2}=0,98\right) ;{ }^{4} \hat{Y}=2,44539+0,00777469 X\left(r^{2}=0,99\right) ;$ $5 \hat{Y}=95,2342+0,324221 X\left(r^{2}=0,99\right) ;{ }^{6} \hat{Y}=5,79254+0,0219322 X\left(r^{2}=0,98\right) ;{ }^{7} \hat{Y}=0,886803+0,00321680 X\left(r^{2}=0,98\right) ;{ }^{8} \hat{Y}=0,177903-0,000100974 X+$ $0,0000221772 * X^{2}\left(R^{2}=0,99\right) ; 9 \hat{Y}=4,73389+0,0177096 X\left(r^{2}=0,98\right) ; 10 \hat{Y}=1,38783-0,00522304 X\left(r^{2}=0,99\right) ; 11 \hat{Y}=54,1003-0,195412 X\left(r^{2}=0,99\right) ;$ $12 \hat{Y}=3,29774-0,01106 X\left(r^{2}=0,99\right) ;{ }^{13} \hat{Y}=5,14071+0,0172947 X\left(r^{2}=0,94\right) ; 14 \hat{Y}=0,0633589+0,0000168591 X+0,00000730953^{*} X^{2}\left(R^{2}=0,79\right) ;$ $15 \hat{\mathrm{Y}}=0,912753+0,00328842 \mathrm{X}\left(\mathrm{r}^{2}=0,98\right)$.

ocorrido aumento na taxa de escape das partículas do rúmen e diminuição no tempo de retenção. Segundo Shaver et al. (1988), elevada proporção de pequenas partículas aumenta a taxa de escape e diminui o tempo de retenção da digesta no rúmen.

Neste estudo, a substituição do feno pela polpa cítrica não alterou o teor de CT. No entanto, ocorreu substituição dos carboidratos fibrosos pelos não-fibrosos (Tabela 2), o que provavelmente alterou a taxa de passagem, contribuindo para o aumento do consumo de MS. Hoover (1986) afirmou que diferentes relações volumoso:concentrado e o uso de subprodutos como polpa cítrica podem alterar a taxa de degradação da parede celular pelos microrganismos ruminais, podendo ser responsáveis, em parte, pelas variações no consumo de matéria seca em animais ruminantes. O consumo de FDN observado neste experimento foi superior à capacidade ótima de consumo de FDN para animais em crescimento, que, segundo Mertens (1985), é de 1\% do PV. O aumento no consumo de MS com a diminuição do teor de FDN das dietas evidencia que o consumo voluntário pode ter sido regulado pela limitação física do trato gastrointestinal. De acordo com Forbes (1986), dietas com elevada capacidade de enchimento inibem a ingestão voluntária por mecanismos que envolvem receptores de tensão localizados na parede do rúmen e retículo, que enviam estímulos para o centro de saciedade do cérebro, determinando interrupção do consumo voluntário de alimentos, o que pode ter ocorrido com as dietas com maior proporção de feno.
Wainman \& Deiwey (1988) observaram restrições severas na ingestão de MS quando a polpa cítrica peletizada passou de $30 \%$ da MS total da dieta. Neste estudo, no tratamento com maior nível de substituição do feno, a polpa cítrica constituía 35\% da MS da dieta e proporcionou aumento no consumo voluntário. As diferenças na relação volumoso:concentrado e na composição das dietas podem explicar a discrepância entre os resultados. As dietas experimentais utilizadas obedeceram às recomendações do NRC (1989) quanto ao mínimo de fibra efetiva, o que também pode ter contribuído para que, mesmo com a polpa cítrica perfazendo $35 \%$ da dieta, houvesse aumento no consumo voluntário.

Os consumos de PB, MO e CT aumentaram com a substituição do feno pela polpa cítrica, o que, provavelmente pode ser explicado pelo aumento na ingestão voluntária de alimentos, uma vez que as dietas eram isoprotéicas e apresentavam teores de MO e CT semelhantes. Foi observado aumento no consumo de NDT, provavelmente em decorrência do maior consumo de MS. O consumo de EE variou de forma quadrática com o nível de substituição do feno pela polpa cítrica, sendo menor quando a polpa substituiu $2,27 \%$ do feno.

$\mathrm{O}$ consumo de $\mathrm{Ca}$ e $\mathrm{P}$ aumentou $(\mathrm{P}<0,10)$ à medida que o feno foi substituído pela polpa cítrica, o que pode ter sido ocasionado pelo aumento no consumo de MS e pelos respectivos teores de $\mathrm{Ca}$ e $\mathrm{P}$ das dietas com maior nível de substituição do feno pela polpa. 
As médias, as equações de regressão e os coeficientes de variação e determinação para os coeficientes de digestibilidade de MS, MO, PB, EE, CT e FDN são descritos na Tabela 4. Com o aumento dos níveis de substituição do feno pela polpa cítrica, foi observada redução linear nas digestibilidades da MS, MO, PB e FDN. Segundo Jaster \& Murphy (1983) e Grant (1997), a redução no percentual de forragem ou a diminuição no tamanho das partículas com o aumento do nível de concentrado resulta em diminuição do $\mathrm{pH}$, da taxa de digestão ruminal e do tempo de retenção, aumento na taxa de passagem e alteração da degradação física e química das partículas, além de mudanças no comportamento ingestivo e de mastigação com conseqüente modificação, entre outros, do tamponamento do ambiente ruminal.

Ressalta-se que, com a substituição do feno pela polpa cítrica, além da mudança física em termos de granulometria das partículas e da densidade, ocorre acentuada mudança química com substituição de parte da FDN por CNF, o que influencia o $\mathrm{pH}$ ruminal, o crescimento microbiano, a taxa de passagem e o tempo de retenção, o que explica, em parte, a redução na digestibilidade da MS, MO, PB e FDN.

Outro fator que pode ter influenciado a digestão da FDN e, conseqüentemente, da MS e MO seria o aumento na quantidade de carboidratos de fácil fermentação, resultando em alterações na microflora ruminal. Coelho \& Leão (1979), Hoover (1986) e Van Soest (1994) afirmaram que a adição de grande quantidade de carboidratos de fácil fermentação pode reduzir a digestibilidade da fração fibrosa e estes efeitos podem ser mais expressivos dependendo da quantidade de $\mathrm{N}-\mathrm{NH}_{3}$ para crescimento microbiano. A substituição do feno pela polpa cítrica reduziu linearmente $(\mathrm{P}<0,01)$ a digestibilidade da proteína.

Alguns autores (Martinez Pascual \& Fernandez Carmona, 1980; Bruno Filho et al., 2000) afirmam que parte do nitrogênio da polpa cítrica está indisponível para o animal na forma de N-FDA, em virtude da reação de Maillard durante seu processamento. A provável menor disponibilidade do nitrogênio das dietas com maior nível de polpa em substituição ao feno pode ter causado redução no crescimento microbiano e, conseqüentemente, na digestibilidade dos nutrientes, principalmente da fibra. Segundo Mcallan \& Smith (1983), a concentração de amônia requerida pelos microrganismos aderentes que digerem fibra é maior que a exigida pelos organismos em suspensão no líquido de rúmen que utilizam carboidratos de fácil digestão. Portanto, em dietas com menor disponibilidade de nitrogênio e maior concentração de carboidratos de rápida fermentação, os organismos celulolíticos podem ter seu crescimento diminuído. Nestas condições, os microrganismos aumentariam seu requerimento de amônia para manter ótimo crescimento microbiano (Hoover, 1986). As dietas experimentais continham o mesmo teor de NT e NNP. No entanto, o aumento da proporção de polpa na dieta provavelmente ocasionou aumento no teor de NIDA, em decorrência do maior teor desta fração na polpa em relação ao feno e ao aumento no teor de CNF (Tabela 2). Estas condições, conforme relatado por Hoover (1986), seriam propícias para diminuição do crescimento dos organismos celulolíticos e da digestibilidade da fibra, colaborando para a menor digestão da MS e MO quando o feno foi substituído pela polpa cítrica.

Não foi observado efeito significativo dos níveis de substituição do feno pela polpa cítrica nas dietas sobre os coeficientes de digestibilidade do EE e dos CT. A variação no teor de EE nas dietas foi muito pequena e a modificação na composição desta fração, causada pela substituição do feno pela polpa cítrica, pode não ter sido suficiente para alterar sua digestibilidade. Com o aumento da substituição do feno pela polpa, os carboidratos fibrosos foram substituídos, em parte, pelos não-fibrosos, que apresentam maior coeficiente de digestibilidade. No entanto, a diminuição na

Tabela 4 - Digestibilidades médias diárias de nutrientes em novilhas alimentadas com dietas contendo polpa cítrica em substituição ao feno de tifton

Table 4 - Apparent total tract digestibilities of nutrients in dairy heifers fed diets containing increasing levels of citrus pulp in replacement of Tifton 85 hay

\begin{tabular}{|c|c|c|c|c|c|c|c|}
\hline \multirow[t]{2}{*}{$\begin{array}{l}\text { Digestibilidade (\%) } \\
\text { Digestibility (\%) }\end{array}$} & \multicolumn{4}{|c|}{$\begin{array}{l}\text { Nível de substituição (\%) } \\
\text { Replacement level (\%) }\end{array}$} & \multirow[t]{2}{*}{$\mathrm{CV}$} & \multicolumn{2}{|c|}{$\mathrm{P}$} \\
\hline & 0 & 16,6 & 33,3 & 50 & & $\mathrm{~L}$ & Q \\
\hline $\operatorname{MS}(D M)^{1}$ & 74,93 & 73,02 & 73,54 & 71,49 & 4,47 & 0,0619 & $\mathrm{~ns}$ \\
\hline $\mathrm{MO}(O M)^{2}$ & 76,39 & 74,54 & 74,93 & 72,94 & 4,20 & 0,0517 & $\mathrm{~ns}$ \\
\hline $\mathrm{PB}(C P)^{3}$ & 74,44 & 71,95 & 68,64 & 65,01 & 5,48 & 0,0001 & $\mathrm{~ns}$ \\
\hline $\mathrm{EE}(E E)^{4}$ & 84,41 & 77,42 & 83,02 & 80,35 & 4,62 & $\mathrm{~ns}$ & $\mathrm{~ns}$ \\
\hline $\mathrm{CT}(T C)^{5}$ & 76,43 & 74,92 & 75,78 & 74,14 & 4,13 & $\mathrm{~ns}$ & $\mathrm{~ns}$ \\
\hline FDN $(N D F)^{6}$ & 71,74 & 67,08 & 64,75 & 58,19 & 6,68 & 0,0001 & $\mathrm{~ns}$ \\
\hline
\end{tabular}

${ }^{1} \hat{\mathrm{Y}}=74,7537-0,059345 \mathrm{X}\left(\mathrm{r}^{2}=0,91\right) ;^{2} \hat{\mathrm{Y}}=76,2299-0,06029 \mathrm{X}\left(\mathrm{r}^{2}=0,92\right) ;{ }^{3} \hat{\mathrm{Y}}=74,7238-0,18922 \mathrm{X}\left(\mathrm{r}^{2}=0,98\right) ;{ }^{4} \hat{\mathrm{Y}}=81,30 ;{ }^{5} \hat{\mathrm{Y}}=75,32 ; 6 \hat{\mathrm{Y}}=71,9082-0,25829 \mathrm{X}$ $\left(r^{2}=0,97\right)$ 
digestão da FDN com aumento do nível de substituição parece ter anulado o efeito positivo da inclusão de CNF nas dietas sobre a digestão dos carboidratos totais.

As médias, as equações de regressão e os coeficientes de variação e determinação para os ganhos de peso (GP), altura de cernelha (AC), altura de garupa (AG), perímetro torácico (PT) e conversão alimentar (CA) estão sumarizados na Tabela 5. Observou-se aumento linear $(\mathrm{P}<0,01)$ no ganho de peso das novilhas à medida que o feno foi substituído pela polpa cítrica. $\mathrm{O}$ aumento no ganho de peso pode ser explicado pelo aumento linear no consumo de MS nos animais alimentados com maior nível de substituição do feno nas dietas. $\mathrm{O}$ ganho de peso foi maior que o predito pelo NRC (1989), ou seja, de $700 \mathrm{~g} /$ dia para o qual as dietas foram ajustadas.

Henrique et al. (1998) não observaram efeito de diferentes níveis de polpa cítrica para novilhas em terminação, sendo o ganho de peso médio de $1,24 \mathrm{~kg} /$ dia. No entanto, as dietas continham diferentes níveis de energia e proteína, o que pode explicar a ausência de efeito da inclusão de polpa cítrica sobre o ganho de peso dos animais.

Velloso (1985) relatou que a polpa cítrica pode ser utilizada em níveis de até $30 \%$ da dieta na alimentação de bovinos. Wainman \& Deivey (1988) afirmaram que níveis superiores a $30 \%$ de polpa cítrica na dieta ocasionam severas reduções no consumo e no desempenho dos animais. Por outro lado, Sampaio et al. (1988) utilizaram dietas com até $56 \%$ de polpa cítrica, com base na MS, para novilhos em confinamento e não observaram redução no consumo e no desempenho dos animais. Os autores relataram ganho de peso médio de $0,95 \mathrm{~kg} / \mathrm{dia}$. As divergências observadas na literatura quanto ao limite de inclusão da polpa cítrica na alimentação de ruminantes são ocasionadas provavelmente pelas diferenças na relação volumoso:concentrado, no teor e na efetividade da fibra, nas características dos alimentos utilizados para compor as dietas e na categoria animal.
A velocidade de crescimento dos animais diminuiu linearmente com o aumento no nível de substituição do feno pela polpa cítrica. $\mathrm{O}$ ganho em $\mathrm{cm} /$ dia de altura de cernelha decresceu linearmente $(\mathrm{P}<0,10)$ e o de altura de garupa não foi afetado pela substituição do feno pela polpa cítrica. $O$ ganho em $\mathrm{cm} /$ dia de perímetro torácico, no entanto, aumentou linearmente $(\mathrm{P}<0,05)$ à medida que $\mathrm{o}$ feno foi substituído pela polpa cítrica.

Os animais alimentados com as dietas contendo maior nível de substituição do feno pela polpa tiveram maior deposição de gordura como resultado do maior ganho de peso, do menor crescimento e do maior aumento no perímetro torácico. Com o aumento da polpa cítrica nas dietas, foi observada diminuição de aproximadamente dez unidades percentuais na digestibilidade da PB quando comparados os tratamentos com 0 e 50\% de substituição do feno. Desta forma, o crescimento dos animais pode ter sido limitado pela proteína da dieta, sendo o excesso de energia convertido em gordura. Radcliff et al. (1997) afirmaram que o elevado ganho de peso para novilhas deve ser acompanhado de crescimento para evitar que fiquem excessivamente gordas, com maior possibilidade de terem partos distócicos.

A conversão alimentar não foi afetada pelos níveis de substituição do feno pela polpa cítrica, sendo o valor médio de 6,99. No entanto, foi observada tendência de os tratamentos com menor nível de substituição terem pior conversão alimentar, o que se deve, provavelmente, ao efeito dos tratamentos experimentais sobre o consumo das dietas.

Os custos com alimentação, receita e margem bruta e o custo por quilograma de ganho de peso são apresentados na Tabela 6. A viabilidade econômica das dietas aumentou com a substituição do feno pela polpa. Apesar de o custo de alimentação aumentar à medida que o feno foi substituído pela polpa cítrica, a receita bruta e a margem bruta aumentaram linearmente $(\mathrm{P}<0,10)$, como conseqüência do maior ganho de peso das novilhas.

Tabela 5 - Médias diárias de ganho de peso (GP), altura de cernelha (AC), altura de garupa (AG), perímetro torácico (PT) e conversão alimentar (CA) em novilhas alimentadas com dietas contendo polpa cítrica em substituição ao feno

Table 5 - Average daily weight gain (WG), wither height (WH), hip height (HH), and heart girth (HG) in dairy heifers fed diets containing increasing levels of citrus pulp in replacement of Tifton 85 hay

\begin{tabular}{|c|c|c|c|c|c|c|c|}
\hline & \multicolumn{4}{|c|}{$\begin{array}{l}\text { Nível de substituição (\%) } \\
\text { Replacement level (\%) }\end{array}$} & \multirow[t]{2}{*}{$\mathrm{CV}$} & \multicolumn{2}{|c|}{$P$} \\
\hline & 0 & 16,6 & 33,3 & 50 & & $\mathrm{~L}$ & $\mathrm{Q}$ \\
\hline $\mathrm{GP}^{1}(\mathrm{~kg} / \mathrm{dia})(W G, k g /$ day $)$ & 0,92 & 1,00 & 1,12 & 1,18 & 13,47 & 0,0018 & $\mathrm{~ns}$ \\
\hline $\mathrm{AC}^{2}(\mathrm{~cm} / \mathrm{dia})(W H, \mathrm{~cm} /$ day $)$ & 0,11 & 0,11 & 0,09 & 0,09 & 21,94 & 0,0692 & $\mathrm{~ns}$ \\
\hline $\mathrm{AG}^{3}(\mathrm{~cm} / \mathrm{dia})(H H, \mathrm{~cm} /$ day $)$ & 0,11 & 0,11 & 0,10 & 0,10 & 19,57 & $\mathrm{~ns}$ & $\mathrm{~ns}$ \\
\hline $\mathrm{PT}^{4}(\mathrm{~cm} / \mathrm{dia})(H G, \mathrm{~cm} /$ day $)$ & 0,18 & 0,19 & 0,21 & 0,22 & 14,15 & 0,0127 & $\mathrm{~ns}$ \\
\hline $\mathrm{CA}^{5}(F G R)$ & 6,98 & 6,61 & 6,16 & 6,38 & 13,93 & $\mathrm{~ns}$ & $\mathrm{~ns}$ \\
\hline
\end{tabular}

$1 \hat{\mathrm{Y}}=0,921730+0,00539726 \mathrm{X}\left(\mathrm{r}^{2}=0,99\right) ;{ }^{2} \hat{\mathrm{Y}}=0,110454-0,000446559 \mathrm{X}\left(\mathrm{r}^{2}=0,90\right) ;{ }^{3} \hat{\mathrm{Y}}=0,11 ;{ }^{4} \hat{\mathrm{Y}}=0,181398+0,00812427 \mathrm{X}\left(\mathrm{r}^{2}=0,99\right) ;{ }^{5} \hat{\mathrm{Y}}=6,38$ 
Tabela 6 - Médias diárias para custo com alimentação, receita e margem bruta e custo por kg de ganho, em função do nível de substituição do feno de tifton pela polpa cítrica ( $X$ em \%)

Table 6 - $\quad$ Average daily gross revenue, gross margin and cost per $\mathrm{kg}$ of weight gain in dairy heifers fed diets containing increasing levels of citrus pulp in replacement of Tifton 85 hay

\begin{tabular}{|c|c|c|c|c|c|c|c|}
\hline & \multicolumn{4}{|c|}{$\begin{array}{l}\text { Nível de substituição (\%) } \\
\text { Replacement level (\%) }\end{array}$} & \multirow[t]{2}{*}{$\mathrm{CV}$} & \multicolumn{2}{|c|}{$\mathrm{P}$} \\
\hline & 0 & 16,6 & 33,3 & 50 & & $\mathrm{~L}$ & Q \\
\hline \multicolumn{8}{|l|}{$\begin{array}{l}\text { A - custo com alimentação } \\
\text { A - Feeding costs }\end{array}$} \\
\hline $\begin{array}{l}\text { Dieta total oferecida }(\mathrm{kg} \text { MN/nov/dia) } \\
\text { Total feed offered }(\mathrm{kg} \text { fresh feed/animal/day) }\end{array}$ & 7,24 & 7,71 & 8,01 & 8,67 & & & \\
\hline $\begin{array}{l}\text { Custo da dieta }(\mathrm{R} \$ / \mathrm{kg} \mathrm{MN}) \\
\text { Concentrate costs }\end{array}$ & 0,26 & 0,25 & 0,24 & 0,22 & & & \\
\hline $\begin{array}{l}\text { Custo alimentação (R } \$ / \text { nov/dia) } \\
\text { Feed costs }\end{array}$ & 1,89 & 1,92 & 1,90 & 1,94 & & & \\
\hline \multicolumn{8}{|l|}{$\begin{array}{l}\text { B - Receita bruta } \\
B \text { - Gross revenue }\end{array}$} \\
\hline $\begin{array}{l}\text { Ganho de peso }(\mathrm{kg} / \mathrm{dia}) \\
\text { Weight gain ( } \mathrm{kg} / \text { day })\end{array}$ & 0,92 & 1,00 & 1,12 & 1,18 & & & \\
\hline $\begin{array}{l}\text { Preço pago ao produtor }(\mathrm{R} \$ / \mathrm{kg}) \\
\text { Price paid to the producer }\end{array}$ & 3,50 & 3,50 & 3,50 & 3,50 & & & \\
\hline $\begin{array}{l}\text { Receita bruta }(\mathrm{R} \$ / \text { nov } / \mathrm{dia})^{1} \\
\text { Gross revenue }\end{array}$ & 3,22 & 3,51 & 3,92 & 4,14 & 13,47 & 0,0785 & $\mathrm{~ns}$ \\
\hline $\begin{array}{l}\mathrm{C}-\text { Margem bruta }(\mathrm{R} \$ / \text { nov } / \mathrm{dia})^{2} \\
C \text { - Gross margin }\end{array}$ & 1,33 & 1,60 & 2,02 & 2,20 & 25,74 & 0,0892 & ns \\
\hline $\begin{array}{l}\text { D - Custo da alimentação por } \mathrm{kg} \text { de ganho de peso } \\
D \text { - Cost per kg of weight gain }\end{array}$ & 2,11 & 1,89 & 1,69 & 1,66 & 14,38 & 0,0641 & $\mathrm{~ns}$ \\
\hline
\end{tabular}

${ }^{1} \hat{Y}=3,22606+0,0188904 X\left(R^{2}=0,99\right) ;{ }^{2} \hat{Y}=1,3352+0,018091 X\left(R^{2}=0,98\right) ;{ }^{3} \hat{Y}=2,07201-0,00936644 X\left(R^{2}=0,91\right)$.

Neste estudo, o custo por quilograma de ganho de peso diminuiu linearmente $(\mathrm{P}<0,10)$ com o aumento dos níveis de substituição. Nas condições em que este estudo foi realizado, a substituição de até $50 \%$ do feno pela polpa cítrica para novilhas leiteiras aumenta a viabilidade econômica da atividade.

\section{Conclusões}

Dependendo da disponibilidade de forragem ou da conveniência econômica da região, a polpa cítrica pode ser utilizada em substituição ao feno de tifton na alimentação de novilhas leiteiras em níveis de até $35 \%$ da MS da dieta como alternativa para o produtor reduzir a idade à primeira cobertura e ao primeiro parto, com menor custo de alimentação, observando-se a necessidade de corrigir o nível de PB das dietas para que o maior ganho de peso dos animais seja acompanhado de uma taxa de crescimento compatível.

\section{Literatura Citada}

BRUNO FILHO, J.R.; BERCHIELLI, T.T.; ANDRADE, P. et al. Digestibilidade da polpa cítrica peletizada na alimentação de bovinos. In: REUNIÃO ANUAL DA SOCIEDADE BRASILEIRA
DE ZOOTECNIA, 37., 2000, Viçosa, MG. Anais... Viçosa, MG: Sociedade Brasileira de Zootecnia, 2000. p.400.

CARVALHO, M.P.; PERES, J.R. Polpa de citrus seca e peletizada na alimentação de bovinos leiteiros. Campinas: CATI, 1995. 8p. (Boletim Técnico, 224).

COELHO DA SILVA, J.F.; LEÃO, M.I. Fundamentos de nutrição dos ruminantes. Piracicaba: Livroceres, 1979. 384p.

CRAWFORT, R.J.; HOOVER, W.H.; KNOWLTON, P.H. Effects of solids and liquid flows on fermentation in continuous culture. I. Dry matter and fiber digestion, VFA production and protozoa numbers. Journal of Animal Science, v. 51 , p.975-982, 1980 .

FEGEROS, K.; ZERVAS, G.; STAMOULI; S. et al. Nutritive value of dried citrus pulp and its effect on milk yield and milk composition of lactating ewes. Journal of Dairy Science, v.78, p.1116-1121, 1995.

FORBES, J.M. The voluntary intake of farm animals. London: Butterworths, 1986. 206p.

GRANT, R.J. Interactions among forages and non forage fiber sources. Journal of Dairy Science, v.80, p.1438-1446, 1997.

HENRIQUE, W.; LEME, P.R.; LANNA, D.P.D. et al. Substituição de amido por pectina com diferentes níveis de concentrado. 1. Desempenho animal e características de carcaça. Revista Brasileira de Zootecnia, v.27, p.1206-1211, 1998.

HOOVER, W.H. Chemical factors involved in ruminal fiber digestion. Journal of Dairy Science, v.69, p.2755-2766, 1986.

JASTER, E.H.; MURPHY, M.R. Effects of varying particle size of forage on digestion and chewing behavior of dairy heifers. Journal of Dairy Science, v.80, p.1438-1446, 1997.

LAMMERS, B.P.; HEINRICHS, A.J. The response of altering the ratio of dietary protein to energy on growth, feed efficiency, and mammary development in rapidly growing prepubertal heifers. Journal of Dairy Science, v.83, p.977-983, 2000 
LICITRA, G.; HERNANDEZ, T.M.; Van SOEST, P.J. Standardization of procedures for nitrogen fractionation of ruminant feeds. Animal Feed Science and Technology, v.57, p.347-358, 1996.

MCALLAN, A.B.; SMITH, R.H. Effects of level of dietary intake and physical form of protein supplement on the digestibilities of different dietary carbohydrates between mouth and abomasum of young steers. Archiv Fur Tierernahrung, v.33, p.633640, 1983

MARTINEZ PASCUAL, J.; FERNANDEZ CARMONA, J. Composition of citrus pulp. Animal Feed Science and Technology, v.5, p.1-10, 1980 .

MERTENS, D.R. Factors influencing feed intake in lactating cows: From theory to application using neutral detergent fiber. In: GA NUTRITION CONFERENCE, 46., 1985, Athens. Proceedings... Athens: University of Georgia, 1985. p.1-18.

NATIONAL RESEARCH COUNCIL - NRC. Nutrient requirements of dairy cattle. 6.ed. Washington, D.C.: Academic Press, 1989. 158p.

NATIONAL RESEARCH COUNCIL - NRC. Nutrient requirements of dairy cattle. 7.ed. Washington, D.C. Academic Press, 2001. 381p.

RADCLIFF, R.P.; VANDEHAAR, M.J.; SKIDMORE, A.L. et al Effects of diet and bovine somatotropin on heifer growth and mammary development. Journal of Dairy Science, v.80, n.9, p.1996-2003, 1997.

ROCHA FILHO, R.R. Efeitos da polpa de cítrus e do milho sobre parâmetros ruminais. Piracicaba: Escola Superior Luiz de Queiroz, 1998. 71p. Dissertação (Mestrado em Agronomia) - Escola Superior Luiz de Queiroz, 1998.

SAMPAIO, A.A.M.; ANDRADE, P.; OLIVEIRA, M.D.S. et al. Uso de rações com diferentes níveis de proteína e fontes de energia na alimentação de bovinos confinados. Fase II. Revista da Sociedade Brasileira de Zootecnia, v.13, p.528-534, 1988.

SEJRSEN, K.; PURUP, S. Influence of prepubertal feeding level on milk yield potential of dairy heifers: A review. Journal of Animal Science, v.75, p.828-835, 1997.
SHAVER, R.D.; NYTED, A.J.; SATTER, L.D et al. Influence of feed intake, forage physical form, and fiber content on particle size of masticated forage, ruminal digesta and feces of dairy cows. Journal of Animal Science, v.71, p.1566-1572, 1988.

SILVA, P.R.C. Análise de alimentos (métodos químicos e biológicos). Viçosa, MG: Universidade Federal de Viçosa, 1990. $165 \mathrm{p}$.

SNIFFEN, C.J.; O'CONNOR, J.D.; Van SOEST, P.J. et al. A net carbohydrate and protein system for evaluating cattle diets: II - Carbohydrate and protein availability. Journal of Animal Science, v.70, p.3562-3577, 1992.

UNIVERSIDADE FEDERAL DE VIÇOSA - UFV. S.A.E.G. (Sistema de Análises Estatísticas e Genéticas). Viçosa, MG: 1995. (Versão 5.0). CD-ROM

Van SOEST, P.J. Nutritional ecology of the ruminant. 2.ed. Ithaca: Cornell, 1994. 476p.

Van SOEST, P.J.; ROBERTSON, J.B.; LEWIS, B.A. Methods for dietary fiber, and nonstarch polysaccharides in relation to animal nutrition. Journal of Dairy Science, v.74, p.3583-3597, 1991.

VELLOSO, L. Uso da polpa cítrica na alimentação animal. Comunicado Científico FMVZ-USP, v.9, p.171-214. 1985.

WAINMAN, E.W.; DEWEY, J.S. Feeding evaluation unit. 5.ed Aberdeen: Rowett Research Institute, 1988. 123p.

WILLIANS, C.H.; DAVID, D.J.; IISMA, O. The determination of chromic oxide in faeces samples by atomic absortion spectrophotometry. Journal Agricultural Science, v.59, p.381-385, 1962 .

WING, J.M. Citrus feedstuffs for dairy cattle. Gainesville: Florida Agricultural Experimental Station, 1982. 25p. (Bulletin 829). 